Al Azhar Buletin of Science Vol.(26) No.1, June, 33-50, 2015,.

\title{
Effect Of Omega(782) Resonance on The Response Functions For d(e, $\left.e^{\prime} \pi^{+}\right)$nn Reaction
}

Mahmoud Tammam ${ }^{[1]}$, Zakaria M. M. Mahmoud ${ }^{[2]}$ and Mohamed S. I. Koubisy ${ }^{[1]}$

${ }^{[1]}$ Department of Physics, Faculty of Science, Al-Azhar University, Assiut Branch, Egypt.

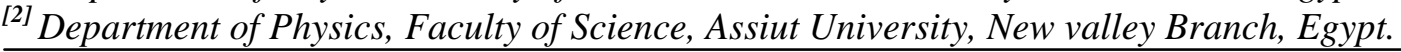

\section{Abstract}

In present work we study the effect of the Omega $(\omega$ (782)) resonance on the response functions for the incoherent positive pion electroproduction forms the deuteron at different values for the four-momentum transfers squared $\left(\mathrm{Q}^{2}\right)$ and the virtual photon lab energy $\left(k_{0}^{l a b}\right)$. The study is carried out in the impulse approximation (IA) i.e. the final state interactions are neglected. The elementary amplitude for pion electroproduction is taken from the MAID-2007 model. The effect seems to be very small but almost increase with increasing the virtual photon lab energy.

Keywords : pion electroproduction; the $\omega$ (782) resonance; Invariant Amplitudes; CGLN amplitudes; Structure functions; the impulse approximation (IA).

PACS numbers: 13.60.Le, 13.40.-f, 21.45.+v, 24.70+s, 25.30.Rw

\section{Introduction}

The pion is now known to exist in three charge states, $\pi^{+}, \pi^{-}$and $\pi^{0}$, with masses of 139.6 $\mathrm{MeV}$ for charged pions and $135.0 \mathrm{MeV}$ for the neutral pion .The Pion photo and electroproduction is presently one of the main sources of our information on the structure of nucleons. With the advent of a new generation of high duty-factor electron accelerators as MAMI (Mainz), ELSA (Bonn), and Jefferson Lab (Newport News) as well as modern laser backscattering facilities as LEGS (Brookhaven) and GRAAL (Grenoble), the photo- and electroproduction of pions on a proton have been studied thoroughly both theoretically and experimentally.

The elementary amplitude of pion photoand electroproduction on free nucleons are one of the main ingredients of the analysis of these reactions for nuclei. In order to study pion electroproduction on complex nuclei, one first has to understand the production process on the nucleon. To this end there have been extensive studies, for example, in [1].

The basic interaction in mesons elctoroproduction is as follows: a virtual photon is incident on a target nucleus and interacts with its constituents. As a result, a pseudoscalar meson is produced along with other particles. Two kinds of processes depending on the nature of the other particles produced in this interaction are found: coherent and incoherent processes. In the coherent process [2,3], the meson is produced with the target nucleus maintaining its initial character. Thus, the interaction starts with a a virtual photon and some nucleus, and ends up with a meson and the same nucleus, i.e $\gamma^{*} \mathrm{XA}-\rightarrow \pi \mathrm{NXA}-1$, where $\mathrm{A}$ is the mass number of the target nucleus. The process is labeled "coherent" because all nucleons in the nucleus participate in the process coherently, leading to a coherent sum of the individual nucleon contributions.

In the incoherent process [4], the nucleus ruptures and thus fails to maintain its initial identity. The meson is produced in association with a nucleon (or an excited state of the nucleon) and some new recoil"daughter" hadronic system. Thus, the interaction starts with a virtual photon and some nucleus and ends up with a meson, a free nucleon (or an excited state of it) and a new hadronic system,i.e. $\gamma^{*} \mathrm{XA}-\rightarrow \pi \mathrm{NXA}-1$. The process is labeled as "incoherent" because it occurs in kinematic and physical circumstances similar to those of the process that produces a meson from a free nucleon.

The pion electroproduction on the deuteron near threshold has been studied in the impulse approximation using an approach based on the unitary transformation method both experimentally $[5,6]$ as well as theoretically [79].

Nucleon resonances are excited states of nucleon particles, often corresponding to one of the quarks having a flipped spin state, or with different orbital angular momentum when the particle decays. The symbol format is given as $\mathrm{N}$ (M)L2I 2J, where $M$ is the particle's approximate mass, $\mathrm{L}$ is the orbital angular momentum of the Nucleon meson pair produced when it decays, and I and $\mathrm{J}$ are the particle's 
isospin and total angular momentum respectively. Since nucleons are defined as having $1 / 2$ isospin, the first number will always be 1 , and the second number will always be odd. When discussing nucleon resonances, sometimes the $\mathrm{N}$ is omitted and the order is reversed, giving L2I 2J(M ). For example, a proton can be symbolized as”N (939) S11” or"S11 (939)”, Delta resonances can be symbolized as" D 33(1232)" and Omega can be symbolized as" $\omega$ (782)"

In this paper the effect of $\omega(782)$ on the semi-exclusive structure functions of the incoherent $\pi^{+}$meson electroproduction off the deuteron is studied at $0.01,0.05$ and $0.1 \mathrm{GeV}^{2}$ four momentum transfer $\left(\mathrm{Q}^{2}\right)$ and different values of the incident virtual photon lab energy $k_{0}^{l a b}$. The present paper is organized as follows; the formalism of $\pi^{+}$electroproduction off the deuteron in the IA is briefly given in section 2 .
The results are summarized and some discussion is presented in section 3. At the end the summary and an outlook are presented.

\section{2-FORMALISM}

The basic formalism for electromagnetic single pion production on the deuteron has been presented in detail for the case of electroproduction [10]. Therefore, we review here only the most important ingredients with due extensions to electroproduction according to the additional contributions from charge and longitudinal current components.

\section{2-1- Kinematics}

The kinematics of the charged pion electroproduction in the one-photon exchange approximation is very similar to photoproduction in replacing the real photon by a virtual one with longitudinal and transverse [11]

$$
\gamma^{*}(k)+d\left(p_{d}\right) \rightarrow n\left(p_{1}\right)+n\left(p_{2}\right)+\pi^{+}(q)
$$

where $K=\left(k_{0}, \vec{k}\right), \quad p_{d}=\left(E_{d}, \vec{d}\right), \quad q=(\omega, \vec{q}), \quad p_{1}=\left(E_{1}, \vec{p}_{1}\right), \quad p_{2}=\left(E_{2}, \overrightarrow{p_{2}}\right)$ denote the fourmomenta of the incoming virtual photon, initial deuteron, the outgoing pion and the two outgoing nucleons, respectively. The energies are given by:

$$
E_{d}=\sqrt{M_{d}+\vec{d}}, \quad E_{1}=\sqrt{M+\overrightarrow{p_{1}}}, \quad E_{2}=\sqrt{M+\overrightarrow{p_{2}}}, \quad \omega=\sqrt{m_{\pi}+\vec{q}}
$$

As coordinate system we choose a righthanded orientation with z-axis along the photon momentum $\vec{q}$ and y-axis perpendicular to the scattering plane along $\overrightarrow{\boldsymbol{k}_{e}} \times \overrightarrow{\boldsymbol{k}_{e}}$. We distinguish in general three planes: (i) the scattering plane spanned by the incoming and scattered electron momenta, (ii) the pion plane, spanned by the photon and pion momenta, which intersects the scattering plane along the z-axis with an angle $\phi_{\pi}$, and (iii) the nucleon plane spanned by the momenta of the two outgoing nucleons intersecting the pion plane along the total momentum of the two nucleons. This is illustrated in Fig. (1)

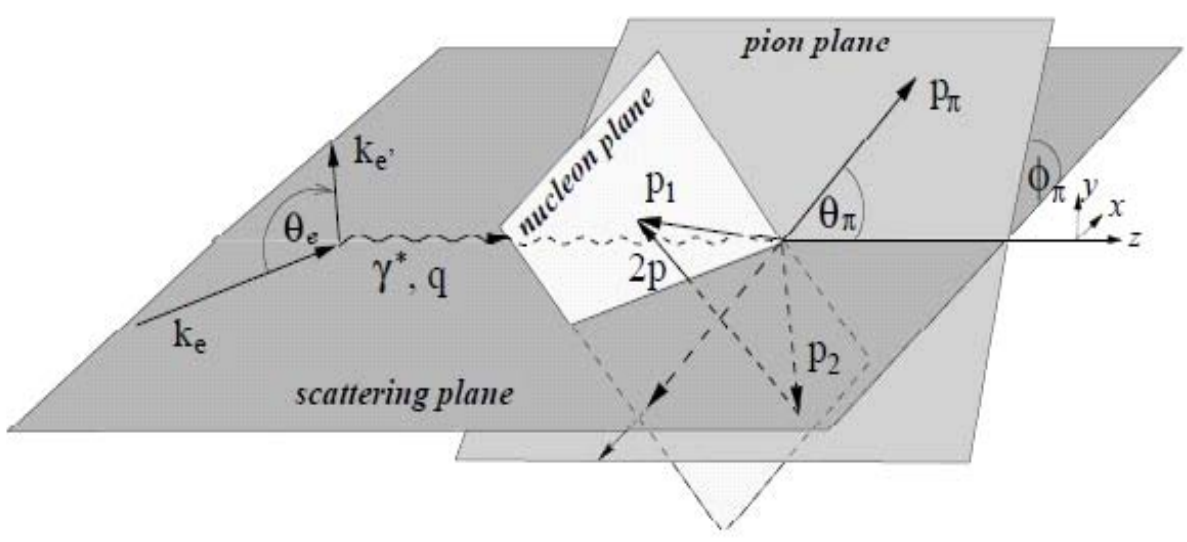

Fig. 1: Kinematics of incoherent single pion electroproduction from the deuteron. 


\section{2-2- The T-matrix}

All observables are determined by the $\mathrm{T}$ matrix elements of the electromagnetic pion production current $J \gamma \pi$ between the initial deuteron and the final $\pi \mathrm{NN}$ states

$$
T_{s m_{s} u m_{d}}=-{ }^{(-)}\left\langle\overrightarrow{p_{1}} \overrightarrow{p_{2}} s m_{s}, \overrightarrow{p_{\pi}}\left|J_{\gamma \pi, \mu}^{(0)}\right| \overrightarrow{p_{d}} 1 m_{d}\right\rangle
$$

Where $\mathrm{s}$ and $\mathrm{m}_{\mathrm{s}}$ denote the total spin and its projection on the relative momentum $\vec{p}$ of the

the deuteron spin projection on the z-axis as quantization axis.

Introducing a partial wave decomposition of the final states, one finds

$$
T_{s m_{s} u m_{d}}\left(W, Q^{2}, p_{\pi}, \Omega_{\pi}, \Omega_{p}\right)=e^{i\left(\mu+m_{d}-m_{s}\right) \varphi_{p \pi}} t_{s m_{s} u m_{d}}\left(W, Q^{2}, p_{\pi}, \theta_{\pi}, \theta_{p}, \varphi_{p \pi}\right)
$$

Where the small t-matrix depends besides in [4] that, if parity is conserved, the following $\mathrm{W}, \mathrm{Q}^{2}$ and $\mathrm{p} \pi$ only on $\theta_{\pi}, \theta_{\mathrm{p}}$ and the relative symmetry relation holds for $\mu= \pm 1$ azimuthal angle $\phi_{\mathrm{p} \pi}=\phi_{\mathrm{p}}-\phi_{\pi}$. We had shown

$$
t_{s-m-s u-m_{d}}\left(W, Q^{2}, p_{\pi}, \theta_{\pi}, \theta_{p}, \varphi_{p \pi}\right)=(-)^{s+\mu+m_{d}+m_{s}} t_{s m_{s} u m_{d}}\left(W, Q^{2}, p_{\pi}, \theta_{\pi}, \theta_{p},-\varphi_{p \pi}\right)
$$

In the present work we include as e.m.current the elementary one-body pion production current of MAID-2007 [12] which has been developed for nuclear applications for photon energies up to $2 \mathrm{GeV}$. It contains Born terms, nucleon resonances P33(1232),

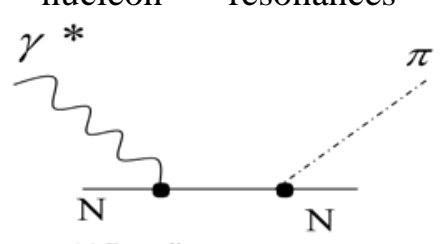

(a) Born direct term

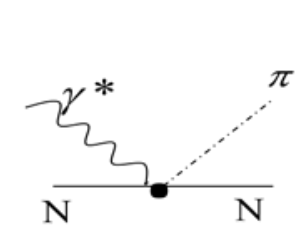

(d) Kroll-Rudermant term

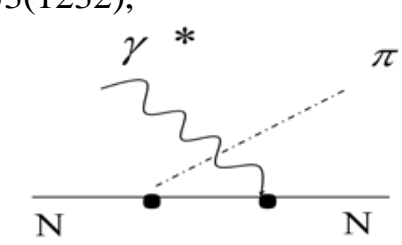

(b) Born crossed term

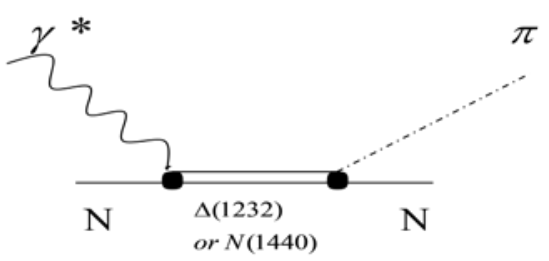

(e) Nucleon resonance exchange (direct)

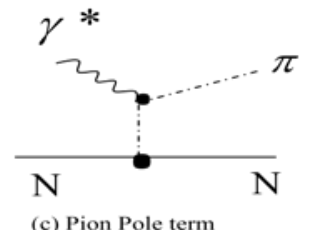

(c) Pion Pole term

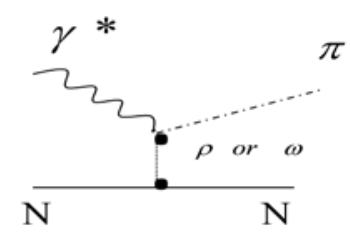

(f) Vector meson t-channel term

Fig. 2: Diagrammatic representation of the elementary pion electroproduction on the nucleon. Born terms: (a)-(d) nucleon, crossed nucleon, pion poles and Kroll-Rudermann contact term; (e): resonance term; (f): vector meson exchange.

For the IA contribution, where the final state with respect to the two outgoing nucleons, one has [13]: is described by a plane wave, antisymmetrized 


$$
\begin{aligned}
T_{s m_{s} \mu m_{d}}^{I A}= & \left\langle\vec{p} s m_{s}, \overrightarrow{p_{\pi}}\left|\left[j_{\gamma \pi, \mu}(1)+j_{\gamma \pi, \mu}(2)\right]\right| 1 m_{d}\right\rangle \\
= & \sqrt{2} \sum_{m_{s}}\left(\langle s m _ { s } | \left\langle\overrightarrow{p_{1}}\left|j_{\gamma \pi, \mu}\left(W_{\gamma N_{1}}, Q^{2}\right)\right|\right.\right. \\
& \left.\times \overrightarrow{p_{d}}-\overrightarrow{p_{2}}\right\rangle \phi_{m_{s}^{\prime} m_{d}}\left(\frac{1}{2} \overrightarrow{p_{d}}-\overrightarrow{p_{2}}\right)\left|1 m_{s}^{\prime}\right\rangle-(1 \leftrightarrow 2)
\end{aligned}
$$

where $\mathrm{J}_{\gamma \pi, \mu}$ denotes the elementary pion photoproduction operator of the MAID-2007 model, $\mathrm{W}_{\gamma \mathrm{N} 1}$ the invariant energy of the $\gamma \mathrm{N} 1$

system, $\overrightarrow{p_{1 / 2}}=\left(\vec{q}+\overrightarrow{p_{d}}-\overrightarrow{p_{\pi}}\right) / 2 \pm \vec{p}$.

$$
\begin{aligned}
\left\langle\vec{p}, 1 m_{s} \mid 1 m_{s}\right\rangle^{(d)} & =\phi_{m_{s} m_{d}}(\vec{p}) \\
& =\sum_{L=0,2} \sum_{m_{l}} i^{L}\left(L m_{L} 1 m_{s} \mid 1 m_{d}\right) u_{L}(p) Y_{L m_{L}}(\hat{p})
\end{aligned}
$$

Furthermore, $\phi_{m_{s} m_{d}}(\vec{p})$ is related to the internal deuteron wave function in momentum space by.

\section{2-3- Cross Section and Structure Functions}

The well-known spectator model in which the pion production takes place on a single nucleon inside the deuteron while the other nucleon acts

$$
\begin{aligned}
\frac{d^{3} \sigma}{d E_{e^{\prime}} d \Omega_{e^{\prime}} d \Omega_{\pi}^{c . m}}= & \frac{\alpha}{(2 \pi)^{2}\left(Q^{2}\right)^{2}} \frac{p_{e^{\prime}}}{p_{e}}\left(\bar{\rho}_{L} R_{L}+\bar{\rho}_{T} R_{T}\right. \\
& \left.+\frac{1}{\sqrt{2}} \bar{\rho}_{L T} R_{L T} \cos \varphi_{\pi}^{c . m}-\bar{\rho}_{T T} R_{T T} \cos 2 \varphi_{\pi}^{c . m}\right)
\end{aligned}
$$

Where the structure functions $\mathrm{R} \alpha(\alpha=\mathrm{L}, \mathrm{T}, \mathrm{LT}, \mathrm{T}$ T $)$ are given in detail by

$$
\begin{aligned}
& R_{L}=W_{00}, \quad R_{T}=W_{11} \\
& R_{L T}=-\sqrt{2} \Re e W_{10} \quad, \quad R_{T T}=W_{1-1} .
\end{aligned}
$$

These structure functions depend on the invariant mass, the squared four momentum transfer $\mathrm{Q}^{2}$, and on the pion angle $\theta_{\pi}^{\text {C.m }}$

\section{3- Results and discussion}

In this section we present and discuss our numerical results for the structure functions of positive and negative pion electroproduction from the deuteron in the IA. As already mentioned, the realistic MAID-2007 model [12] has been used for the evaluation of the as a pure spectator is used to produce the matrix element of electroproduction off the deuteron.

The final expression for the semi-exclusive differential cross section is defined in Ref. [11], and the reader is referred to this work for full details of the next expressions. 
numerical evaluation is based on a Gauss integration for the calculation of the matrix element of the MAID operator using for the deuteron wave function an analytical parameterization of the $\mathrm{S}$ - and $\mathrm{D}$-waves of the Bonn potential in momentum space [14].

In Figs. (3-11) the angular distribution for the four structure functions at different values for the four momentum transfer $\mathrm{Q}^{2}$ and the virtual photon lab energy $k_{0}^{l a b}$. The dotted lines indicate the situation when the $\omega$ (782) resonance contributions are eliminated from the elementary process and the continues ones are when this contributions are added.

It is clear that the bigger contributions come from the longitudinal $\left(\mathrm{R}_{\mathrm{L}}\right)$ and transferee $\left(\mathrm{R}_{\mathrm{T}}\right)$ structure functions.
In Fig.(3), the four momentum transfer $Q^{2}=$ $0.01 \mathrm{GeV} 2$ and the virtual photon lab energy $k_{0}^{l a b}=300 \mathrm{Me}$, the contributions of $\omega(782)$ are not noticeable for all structure functions $R_{L}, R_{T}$, $\mathrm{R}_{\mathrm{TT}}$ and $\mathrm{R}_{\mathrm{LT}}$.

Increasing virtual photon lab energy to $k_{0}^{l a b}=350 \mathrm{MeV}$ and keeping the four momentum transfer $\mathrm{Q}^{2}=0.01 \mathrm{GeV}^{2}$ (Fig.4), the contributions of $\omega(782)$ are very small, for $R_{L}$ still so very small, $\mathrm{R}_{\mathrm{T}}$ resulting in small increase at the forward angles and small decrease at the backward ones, $\mathrm{R}_{\mathrm{LT}}$ a small decrease at the forward and backward angles and a small effect was found for $\mathrm{R}_{\mathrm{T} \text { T }}$ near the peak region.
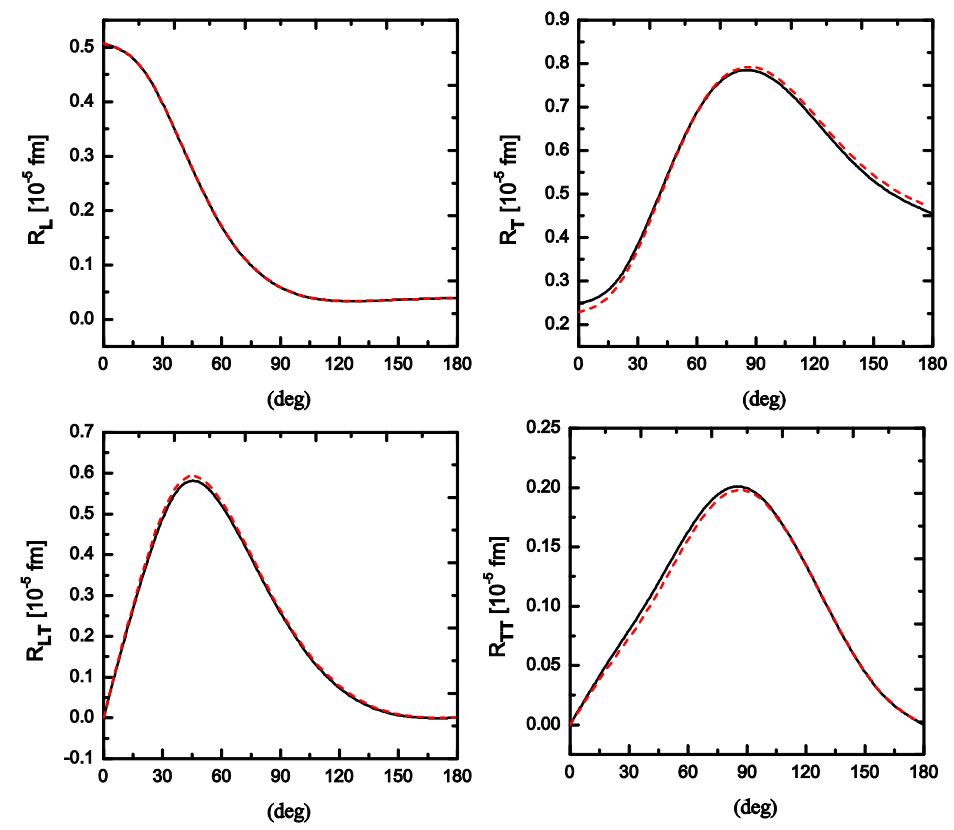

Fig. 3: Angular dependence of the four semi-exclusive structure functions of $d\left(e, e^{\prime} \pi^{+}\right) n n$ at $\mathrm{k}_{0}^{\mathrm{lab}}=300$ $\mathrm{MeV}$ and squared four-momentum transfer $\mathrm{Q}^{2}=0.01(\mathrm{GeV}) 2$, full lines where the $\omega(782)$ is included and dashed lines where $\omega(782)$ is eliminated 

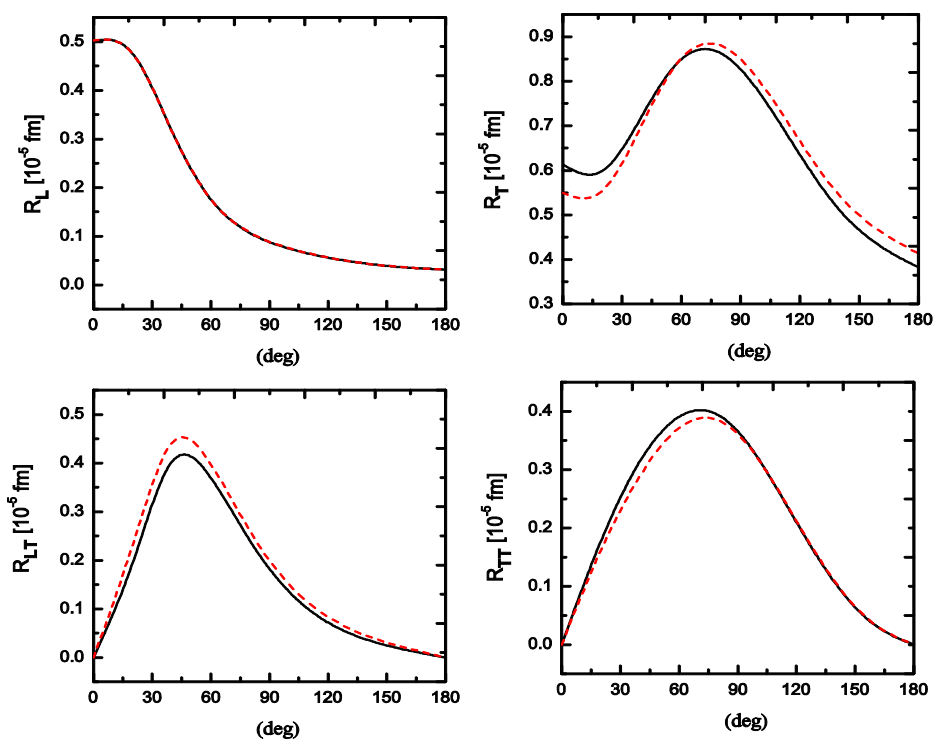

Fig. 4: Notation as in Fig. (3) at $k_{0}^{l a b}=350 \mathrm{MeV}$

In Fig. (5) the virtual photon lab energy is increased to $k_{0}^{l a b}=400 \mathrm{MeV}$ at the same value of the four momentum transfer $\mathrm{Q}^{2}=0.01 \mathrm{GeV}^{2}$, the contributions of $\omega$ (782) are somewhat bigger than what was found in Fig.(4) but still so very small.
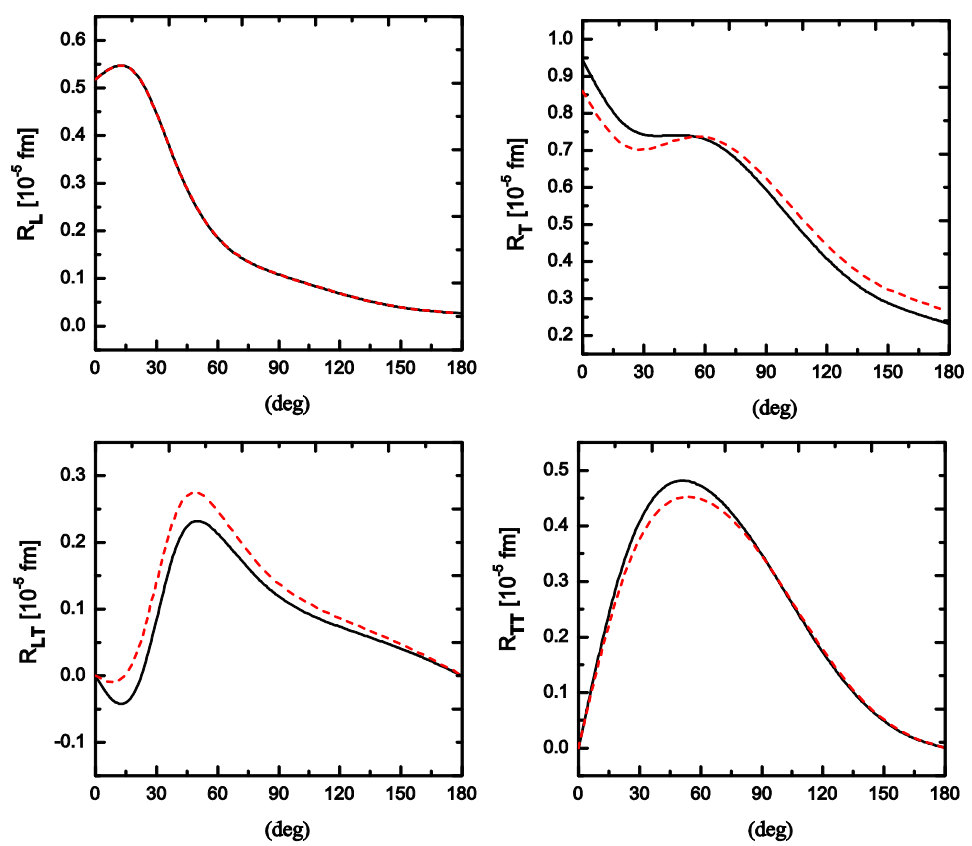

Fig. 5: Notation as in Fig. (3) at $k_{0}^{l a b}=400 \mathrm{MeV}$. 

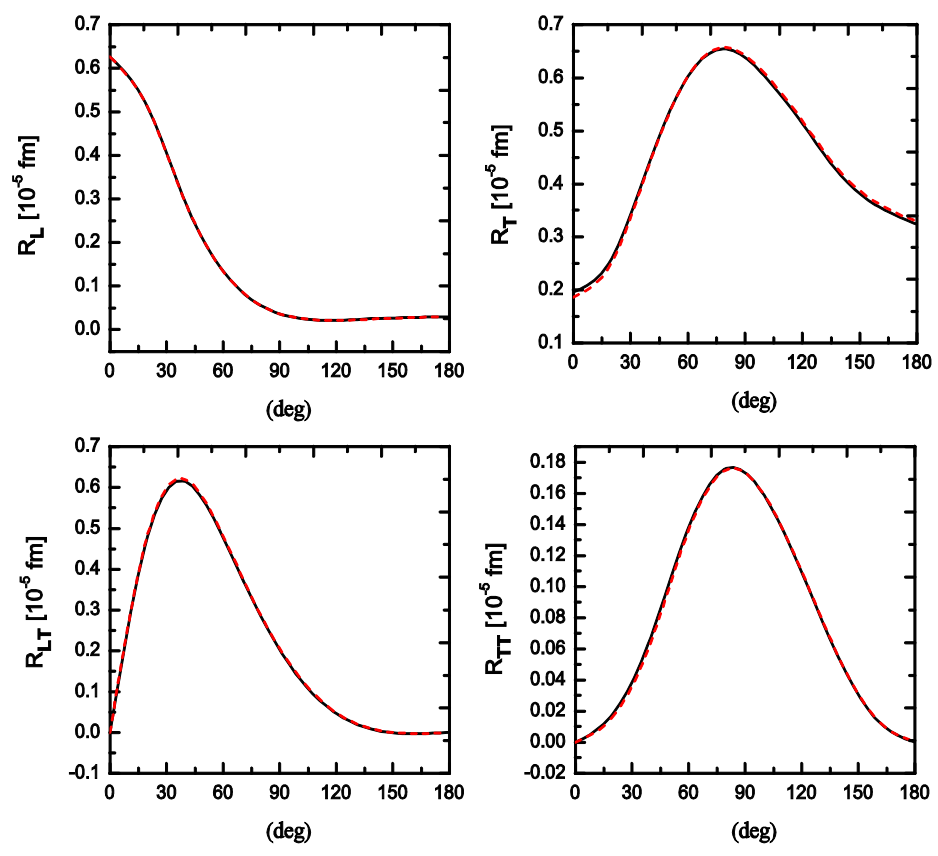

Fig. 6: Notation as in Fig. (3) at $\mathrm{Q}^{2}=0.05 \mathrm{MeV}$..

In Fig.(6) where the four momentum transfer $\mathrm{Q}^{2}=0.05 \mathrm{GeV}^{2}$ and the virtual photon lab energy $k_{0}^{l a b}=300 \mathrm{MeV}$, the contribution of $\omega(782)$ can be neglected for all structure functions.
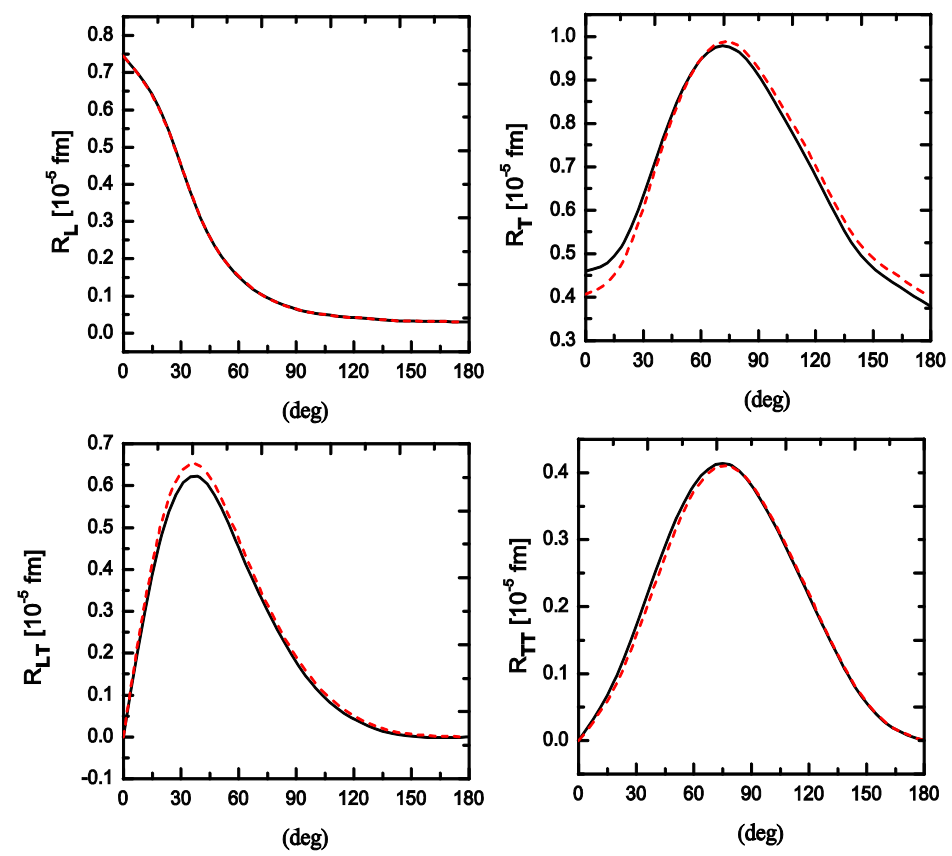

Fig.7: Notation as in Fig. (6) at $\mathrm{k}_{0}^{\mathrm{lab}}=350 \mathrm{MeV}$. 

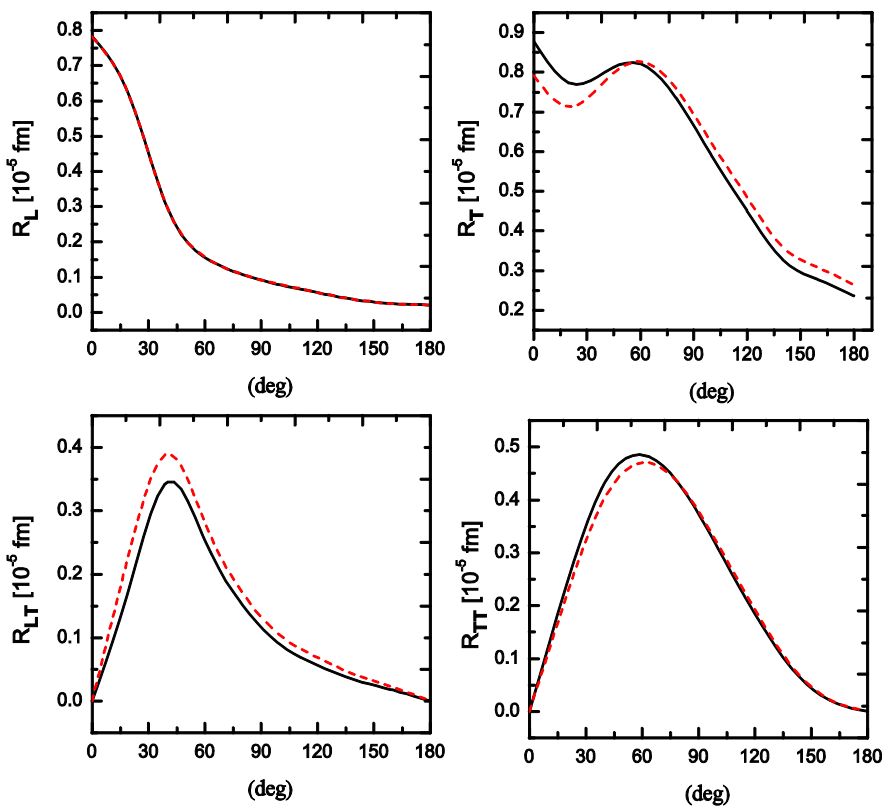

Fig. 8: Notation as in Fig. (6) at $\boldsymbol{k}_{0}^{\mathrm{lab}}=400 \mathrm{MeV}$.

In Fig. (8) the virtual photon lab energy is increased to $k_{0}^{l a b}=400 \mathrm{MeV}$ at the same value of the four momentum transfer $\mathrm{Q}^{2}=0.05 \mathrm{GeV}^{2}$, the contributions of $\omega(782)$ are somewhat bigger than what was found in Fig.(7) but also still so very small.
The same results are found for $\mathrm{Q}^{2}=0.1 \mathrm{GeV}^{2}$ at $k_{0}^{l a b}=300,350$ and $400 \mathrm{MeV}$ (see Figs. 9-11), This mean, the effect of adding $\omega(782)$ is very small and slightly increase with increasing the virtual photon lab energy. The four momentum transferee value has no role in the effect of adding $\omega(782)$ resonance.
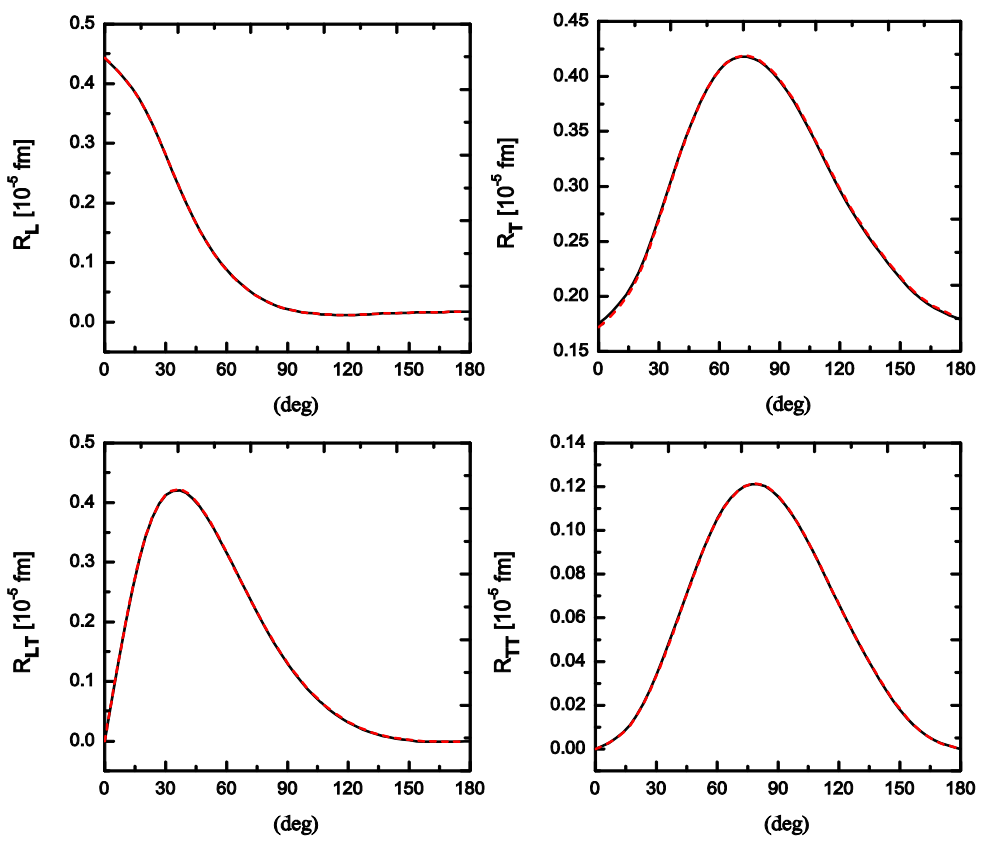

Fig. 9: : Notation as in Fig. (3) at $\mathrm{Q}^{2}=0.1(\mathrm{GeV})^{2}$. 

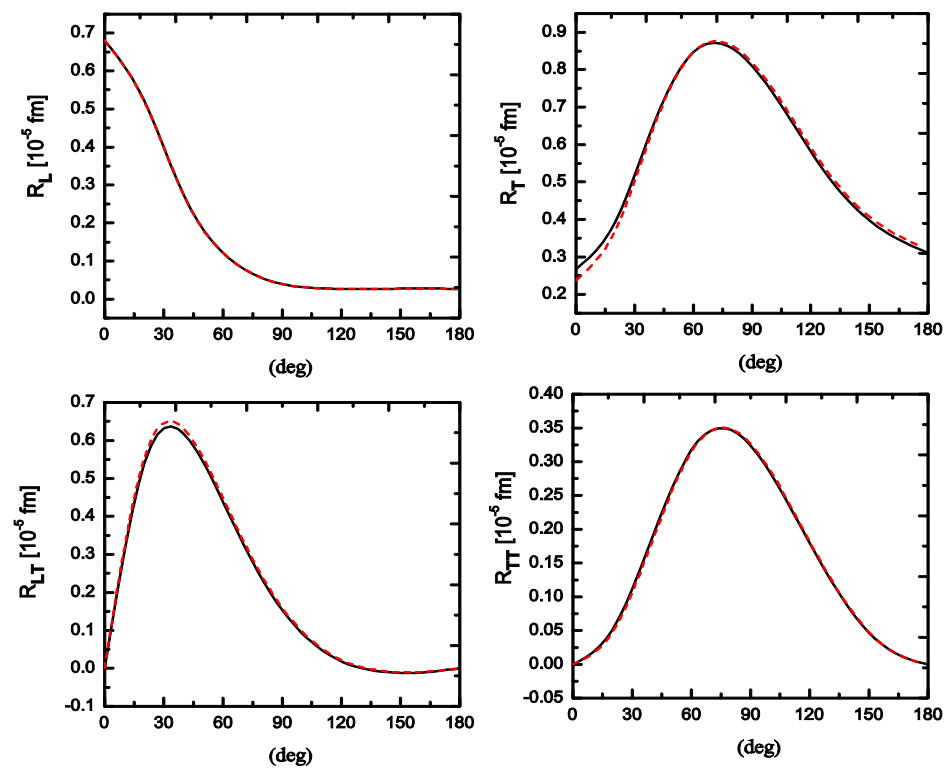

Fig. 10: Notation as in Fig. (9) at $\mathrm{k}_{0}^{\mathrm{lab}}=350 \mathrm{MeV}$.
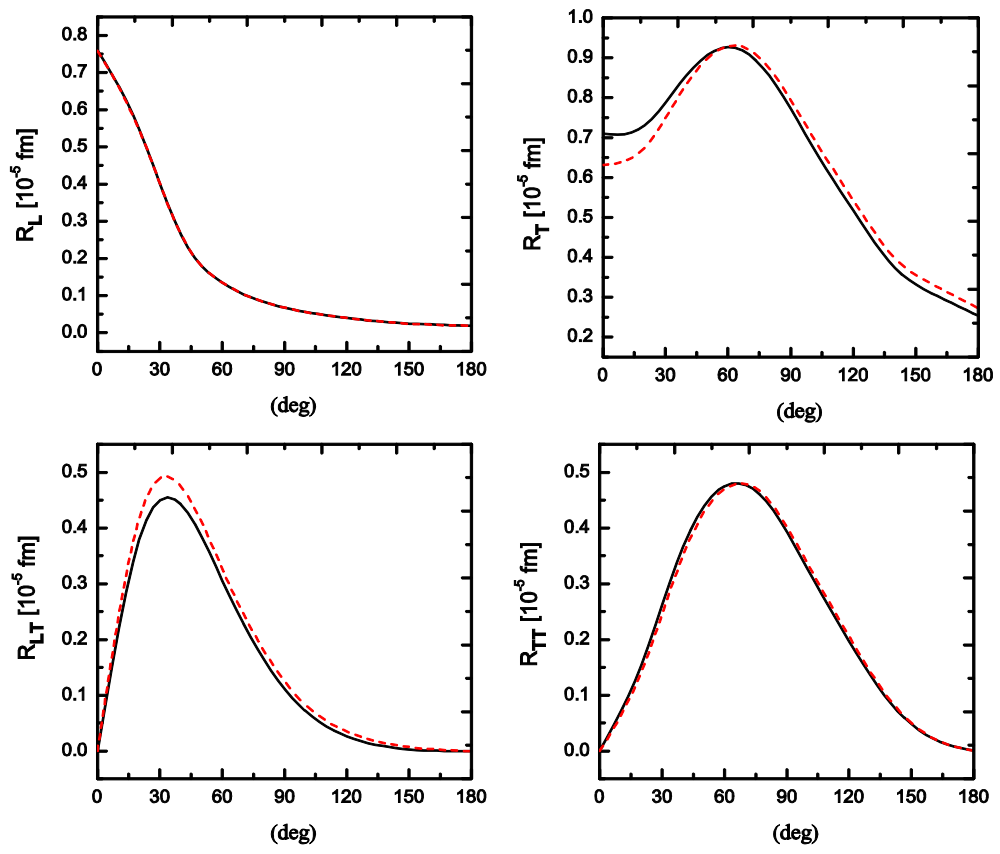

Fig. 11: Notation as in Fig. (9) at $\mathrm{k}_{0}^{\mathrm{lab}}=400 \mathrm{MeV}$.

\section{4- Conclusion}

A systematic study for the contribution of $\omega(782)$ resonance on the structure functions of the positive pion electroproduction of the deuteron is made. This study is made in the IA without adding final state interactions, since the structure functions depend on the squared four momentum transfer $\mathrm{Q}^{2}$, the invariant energy or equivalently the virtual photon laboratory energy and the outgoing pion angle in the final hadronic c.m. system, three values for $\mathrm{Q}^{2}$ and $k_{0}^{\text {lab }}$ have been selected for the presentation of the results. 
The results show a small effect of $\omega$ (782) resonance on the positive pion electroproduction from the deuteron, this effect does not affected by increasing $\mathrm{Q}^{2}$ and increase by increasing $k_{0}^{l a b}$

\section{5- References}

[1] X. Li, PhD dissertation, 1993.ChineseAcademy of Sciences Institute of Physics.

[2] M P.Rekalo, E. Tomasi-Gustafsson and J.Arvieux, Annals of Physics 295, 1 U32 (2002).

[3] E. Breitmoser, H. Arenhövel, Nuclear Physics (A), 612,321-345 (1997) .

[4] A. Fix, H. Arenhövel, Physics Letters (B) 492,32-38 (2000) .

[5] R Gilman, M Bernheim, M Brussel, J Cheminaud, J Danel, J Didelez, M Duval, GFournier, R Frascaria, RJ Holt, HE Jackson,J Kim, E Kinney, J LeGoff, Physical ReviewLetters 64, 622-624 (1990).

[6] D. Gaskell et al., Physical Review Letters 87,202301/15 (2001).

[7] R.J. Loucks, V.R. Pandharipande, and R.Schiavilla, Physical Review (C) 49, 342(1994).

[8] K. Hafidi and T.-S.H. Lee, Physical Review(C) 64, 064607 (2001).

[9] L.G. Levchuk and A.V. Shebeko,NuclearPhysics (A) 62,228-236 (1999).

[10] H. Arenhövel and A. Fix, Physical Review(C) 72, 064004 (2005).

[11] Mahmoud Tammam, A. Fix, H. Arenhövel, Physical Review (C) 74 ,044001(2006).

[12] D. Drechsel, O. Hanstein, S. Kamlov, L. Tiator, Nuclear Physics (A)645,423-438 (1999).

[13] T.W. Donnelly and A.S. Raskin,Annals of Physics (N.Y.) 169, 247 (1986).

[1ح4] Mahmoud Tammam , Nuclear Physics

(A)829,126"U135(2009). 